\title{
EXPERIENCE OF LMCA ANGIOPLASTY FROM A REFERRAL CENTER AND IN HOSPITAL OUTCOMES
}

\author{
P. Siva Satya Subramanyam, P. Sireesha
}

\begin{abstract}
:
Introduction and Objectives: CABG is the treatment of choice for severe left main coronary artery stenosis. The results of a number of multicenter trials have suggested angioplasty with stenting as a possible alternative treatment. Previous studies demonstrated procedural success of 90-100\% and Mortality 0.8-2\% So, want to see these parameters in Asian population though the LMCA registry of a referral center institute.

Material and Methods: We have collected data from patient records who underwent LMCA angioplasty procedure (both unprotected or protected LMCA) performed at our institution between April 2003 to July 2015.Detailed in-hospital data including age, gender, coronary risk factors, procedural events were obtained and analyzed.

Results: Angiographically documented success was obtained in $98 \%$ patients with zero mortality. In 134 patients the lesions were pre-dilated using a conventional balloon. In 19 patients direct stenting was done. The median diameter and length of the stent was $3.2542 \mathrm{~mm}$ and $13.790 \mathrm{~mm}$, respectively. POBA was done in two cases, BMS were used in 27 cases ,DES were used in 124 cases.

Only LMCA stenting was done in 53 cases LMCA with other vessels stenting was done in 98 cases. FFR was used in 2 cases, Kissing balloon dilatation was used in 8 cases, ostial flaring was done in 5 cases. There was no mortality in the study but on table stent thrombosis was seen in one patient for which thrombuster was used, IABP was placed immediately and sent for CABG. For one case result was suboptimal.

Conclusions: Present study demonstrated that stenting for significant LMCA disease is safe and feasible with very high procedural and clinical success rates and zero mortality. LMCA-left main coronary artery, POBA-plain old balloon angioplasty, PCI-Percutaneous coronary intervention, CABGcoronary artery bypass grafting, FFR-Fractional flow reserve, TIMI- thrombolysis in myocardial infarction, DES-drug eluting stent, BMS-bare metal stent, TLR-Target lesion revascularization.

Keywords: Left main disease, PCI, Out comes.
\end{abstract}

Article received on 03 OCT 2016, published on 31 OCT 2016.

P. Siva Satya Subramanyam ${ }^{1}$, P. Sireesha ${ }^{2}$

1 DM student, Department of Cardiology, NIMS, India

2 Assistant Professor, Kamineni Institute of Medical Sciences

Corresponding author: P. Siva Satya Subramanyam,

Email: dr.pss99@gmail.com

\section{INTRODUCTION}

Significant left main coronary artery (LMCA) disease $(>50 \%$ stenosis) is detected during cardiac catheterization in $3 \%-5 \%$ of patients. The recommended optional treatment is coronary artery bypass grafting, which has been shown to improve survival in affected patients [1]. In most centers, percutaneous coronary intervention has traditionally been restricted to patients with high surgical risk or who underwent previous CABG (protected LMCA disease); PCI may also be used as a salvage procedure in cases of angioplasty complications [2].The results depend on the clinical background and the baseline left ventricular function. The introduction of coronary stents renewed interest in the use of PCI for the treatment of LMCA disease. Several case series of patients who were treated electively even though they were at low risk for coronary artery bypass grafting(CABG) reported a nearzero procedural mortality rate and a $3.1 \%$ to $4.2 \%$ one year mortality rate. $[3-5,24]$. With the use of bare metal stents, restenosis remained a significant concern. However in the past few years there have been significant improvements in stent implantation techniques [7], stent technology and post stent antithrombotic agents [8], which have allowed evaluation of the role of PCI for LM disease and dramatic decrease in restenosis rates.

Recent studies have focused on the safety and efficacy of stenting the LMCA to determine whether it does provide a true alternative to coronary artery bypass grafting( $\mathrm{CABG}$ ). Though CABG is the gold standard treatment in case of critical LM disease, recent guidelines have reevaluated the indication of Percutaneous coronary intervention ( $\mathrm{PCI}$ ), suggesting LM PCI can be the safe option in comparison to CABG in selected patient group where high surgical risk \& comorbid conditions are present. 


\section{MATERIALS \& METHODS}

We have collected data from patients who underwent LMCA angioplasty procedure performed at our institution between April 2003 to July 2015. The study sample included patients with both protected and unprotected LMCA stenosis presented with symptoms of the stable and unstable coronary artery disease who were treated with the catheter based approach at Nizam's Institute of Medical Sciences, Hyderabad. The data regarding history physical examination, and investigations was collected from database at the center. Demographic and clinical characteristics of patients were documented. All the patients were classified according to the angiographic profile as unprotected Left main (LM), protected LM , only LM, LM with one vessel disease, LM with two vessel disease and LM with three vessel disease. Angioplasty was performed electively in all patients. Routine transradial or femoral arterial approach was adopted in each patient for Left main(LM) Percutaneous coronary intervention / stenting using 5- French, 6-French (F) or 7-F guiding catheter dependent on then LM lesion character and the strategy of stent implantation.

Unprotected LMCA disease was defined as $\geq 50 \%$ left main stenosis in the absence of a patent coronary graft to one of the major branches of the left coronary artery. Procedures were performed according to the clinical indications. The decision to perform coronary artery bypass grafting or PCI was left to the discretion of the physician, based on the clinical findings, patient's age, co-morbidities, and SYNTAX score. We excluded patients with significant renal, hepatic \& other systemic dysfunction and patients who had cardiogenic shock .

PCI was performed by the conventional technique (dilatation before stenting) or with rotational atherectomy before stenting (in cases of heavy calcification), or by direct stenting, at the discretion of the operator with consideration of the lesion's location and morphology. For tougher lesions multiple exchanges of guide wires were done. Pre dilatation before stent implantation was performed with undersized, conventional angioplasty balloons. The stent was then deployed by inflating the stent delivery balloon at nominal pressure. After deployment of the stent, stented segment were further dilated with high pressure balloon inflation, to achieve angiographic optimization. The balloon inflation were brief $(<60 \mathrm{sec})$ to avoid prolonged ischemia \& ischemia related complications. Additionally, PCI was done to other vessels with significant obstruction that limited the blood flow and eligible for PCI at the same stage or during hospitalization.

The technique for bifurcation lesions included stenting of the left main artery into a major branch of the left coronary artery with or without stenting of the other branch, depending on the degree of residual stenosis of the other branch (e.g., left anterior descending or left circumflex). Kissing balloon inflation was used as the final step of the procedure in cases of bifurcation lesions. The selection of a drug-eluting stent or bare metal stent was done under operator's discretion. Data regarding insertion of intra-aortic balloon pump was included from those patients who received under emergency basis but was not inserted prophylactically. Procedure success was defined a final residual stenosis of less than $10 \%$ and normal blood flow was achieved in the LM coronary artery. The feasibility was defined as failed procedure rate of less than $1.0 \%$. Safety was defined as rate of procedure-related major complication (i.e., failed procedure rate and procedure-related mortality) less than $1.0 \%$

\section{STATISTICAL ANALYSIS:}

Data were expressed as mean $\pm \mathrm{SD}$ or \% (number). Continue data which were expressed as mean \pm SD were compared using one way ANOVA and followed by Bonferroni multiple-comparison post hoc test. Categorical data which were expressed as \% (n) were analyzed by $x 2$ test and followed by Bonferroni multiple comparison post hoc tests. Statistical analysis was performed using SAS statistical software for Windows version 8.2 (SAS institute, Cary, NC). P value of $<0.05$ was considered statistically significant.

\section{RESULTS}

We have analyzed data of 153 patients with significant LMCA disease presented with ACS and CSA who underwent PCI from our tertiary care center in South India between April 2003 to July 2015. The baseline characteristics, clinical presentation, angiographic profile were given in Table 1.

Out of total 153 patients males were 114 (74.5\%), females were $39(25.5 \%)$, with mean age of presentation in males was $57.9 \pm 9.7$ years and in females was $58.7 \pm$ 
12.3 years without statistical difference $(p=0.7) .18$ patients $(11.8 \%)$ were diabetics and 22 patients (14.4\%) were hypertensives with no significant $\mathrm{P}$ value. LV dysfunction was noted in $15(38.5 \%)$ in females with Severe being $6(15.4 \%)$ and in males $28(24.6 \%)$ with Severe $7(6.1 \%)$ with no statistical significance.

Table 1 : Baseline characteristics, clinical presentation, angiographic profile of patients

\begin{tabular}{|l|l|l|l|}
\hline Parameter & Female & Male & $\begin{array}{l}\text { P } \\
\text { VALUE }\end{array}$ \\
\hline Total No & $39(25.5 \%)$ & $114(74.5 \%)$ & \\
\hline Age & $58.7 \pm 12.3$ & $57.9 \pm 9.7$ & 0.7 \\
\hline HTN & $6(15.4 \%)$ & $16(14 \%)$ & 0.8 \\
\hline DM & $6(15.4 \%)$ & $12(10.5 \%)$ & 0.5 \\
\hline LV Dysfunction & $15(38.5 \%)$ & $28(24.6 \%)$ & 0.11 \\
\hline Mild & $4(10.3 \%)$ & $15(13.2 \%)$ & 0.6 \\
\hline Moderate & $5(12.8 \%)$ & $6(5.3 \%)$ & 0.2 \\
\hline Severe & $6(15.4 \%)$ & $7(6.1 \%)$ & 0.13 \\
\hline $\begin{array}{l}\text { Type of presentation } \\
\text { (ACS) }\end{array}$ & $16(25.4 \%)$ & $47(74.6 \%)$ & 0.05 \\
\hline Protected LMCA & $8(20.5 \%)$ & $40(35.96 \%)$ & 0.05 \\
\hline Only LMCA & $16(41 \%)$ & $39(34.2 \%)$ & 0.5 \\
\hline LMCA+-One vessel & $13(33.3 \%)$ & $51(44.7 \%)$ & 0.2 \\
\hline LMCA-+Two vessel & $9(23.1 \%)$ & $18(15.8 \%)$ & 0.4 \\
\hline LMCA+-Three vessel & $1(2.6 \%)$ & $6(5.3 \%)$ & 0.4 \\
\hline Dultiple wires & $2(5.1 \%)$ & $6(5.3 \%)$ & 0.97 \\
\hline Decond generation & $35(89.7 \%)$ & $89(78.1 \%)$ & 0.6 \\
\hline
\end{tabular}

Fig 1: Distribution of LMCA disease.

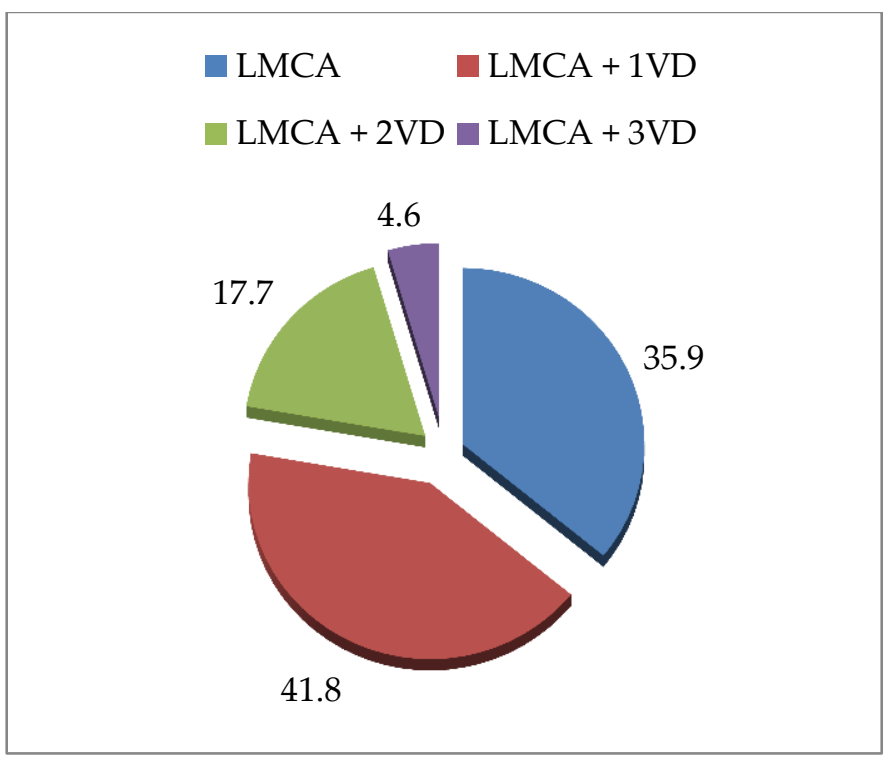

Patients were classified under the mentioned headings as Protected LMCA, unprotected LMCA, Only LMCA, LMCA+One vessel, LMCA+Two vessel, LMCA+Three vessel (Fig1).

Out of total 153 cases there are 105 unprotected LMCA cases, and 48 are protected LMCA cases. Out of 105 unprotected LMCA cases, 44 cases had only LMCA disease (Table 1).

Out of 48 protected LMCA cases, 11 cases had only LMCA disease, 25 cases had LMCA+-One vessel disease, 9 cases had LMCA+Two vessel, 3 cases had LMCA+Three vessel disease. There is no significant $\mathrm{P}$ value among the above mentioned groups except between males and females under Protected LMCA group $(P$ value $=0.05)$. Patients with significant left main disease who presented with ACS are 63, out of which 16(25.4\%)are females and 47(74.6\%)are males and 90 patients presented with CSA, out of which 23(25.5\%)are females and $67(74.5 \%)$ are females (Table 1 ).

Table 2 : Classification of LMCA lesions

\begin{tabular}{|l|l|l|l|}
\hline $\begin{array}{l}\text { Pattern of LM } \\
\text { involvement }\end{array}$ & Total & Female & Male \\
\hline Ostial & $32(20.9 \%)$ & $7(21.8 \%)$ & $25(78.1 \%)$ \\
\hline Mid shaft & $65(42 \%)$ & $25(38.4 \%)$ & $40(61.5 \%)$ \\
\hline Distal & $56(36 \%)$ & $8(14.2 \%)$ & $48(85.7 \%)$ \\
\hline
\end{tabular}


The stenotic LMCA lesions were located in the ostium in $32(20.9 \%)$ females being $7(21.8 \%)$ males $25(78.1 \%)$, in the shaft in $65(42 \%)$ females being $25(38.4 \%)$, males $40(61.5 \%)$ and distally in $56(36 \%)$ females being 78 (14.2\%) and males $48(85.7 \%)$ respectively (Table 2$)$.

Table 3: Guide wires and guide catheters

\begin{tabular}{|c|c|c|c|}
\hline \multicolumn{2}{|l|}{ Guides } & \multicolumn{2}{|l|}{ Wires } \\
\hline Variable & No & Variable & $\begin{array}{l}\mathbf{N} \\
\mathbf{0}\end{array}$ \\
\hline JL 3 & 6 & All Star & 1 \\
\hline XB 3.5 & 18 & ATW & 15 \\
\hline AL 3.5 & 1 & BMW & 1 \\
\hline EBU 4 & 1 & Choice extra support & 4 \\
\hline EBU 3 & 8 & Choice floppy & 29 \\
\hline EBU 3.5 & 28 & Choice intermediate & 1 \\
\hline JL 3.5 & 86 & Choice PT & 1 \\
\hline JL 4 & 5 & CougarXT & 28 \\
\hline \multicolumn{2}{|l|}{ Extra Wires } & Cross it & 3 \\
\hline AL2 & 1 & Fielder XT & 1 \\
\hline XB3.5 & 3 & FFR & 3 \\
\hline EBU3.5 & 1 & Miracle & 1 \\
\hline JL3.5 with SH & 1 & Stabilizer & 43 \\
\hline JL 4 & 1 & Sion blue & 1 \\
\hline \multirow[t]{4}{*}{ JL3 } & 1 & Whisper & 1 \\
\hline & & Ginger light & 3 \\
\hline & & Ginger Medium & 4 \\
\hline & & Ginger Support & 13 \\
\hline
\end{tabular}

$6 \mathrm{~F}$ catheter was used in $119(77.8), 7 \mathrm{~F}$ catheter was used in $33(21.6 \%)$ and $5 \mathrm{~F}$ catheter was used in $1(0.6 \%)$. In eight patients we have used more than one guide for engagement (AL2-1, XB3.5-3, EBU3.5-1, JL3.5 WITH SH1,JL 4-1,JL3-1) and in 16 patients we have used more than one wire for the procedure (Cougar XT -2, Sion blue -2, Fielder XT-1, Stabilizer Plus -5, ATW -1, Ginger 1, Ginger support ). Types of wires and guides used were given in the Table 3 . Most of the cases were engaged with JL 3.5(86 cases), EBU 3.5 (28 cases) , XB 3.5 (18 cases).

Stabilizer wire was used in 43 cases, Choice floppy was used in 29 cases, Cougar XT was used in 28 cases, ATW was used in 15 cases and Ginger Support was used in 13 cases without statistical significance (Table 3).
In 134 patients $(87.5 \%)$, the lesions were pre-dilated using a conventional balloon catheter whose diameter was less than that of the reference segment and which was inflated to the pressure needed to obtain the required degree of distention. In 19 patients (12.4\%), direct stenting was done. The stent position had been confirmed by angiography, the balloon was inflated for up to max of $60 \mathrm{~s}$. The maximum inflation pressure was 12 \pm 2 Atm (range, 9-20 Atm). The median diameter and length of the stent was $3.2542 \mathrm{~mm}$ and $13.790 \mathrm{~mm}$, respectively. After stent deployment, at least 2 orthogonal images were obtained to verify the result achieved. In cases in which results were considered to be suboptimal, further dilatation was carried out using the same balloon at a higher inflation pressure or using a larger diameter, less distensible balloon inflated at a high pressure.

POBA was done in two cases $2(1.7 \%)$, BMS were used in 27 cases $(17.64 \%)$, DES were used in 124 cases first generation $-37(24.1 \%)$, second generation $-87(56.8 \%)$ (Table 1).

Table 4: Angiographic Characteristics

\begin{tabular}{|l|c|}
\hline Description of LMCA disease & No (\%) \\
\hline Critical isolated LM disease & $55(35.94 \%)$ \\
\hline Critical LM with other vessels disease & $98(64 \%)$ \\
\hline Distal LM & $56(36 \%)$ \\
\hline Distal LM and ostial LAD & $16(10.4 \%)$ \\
\hline Distal LM and ostial LCX & $30(19.6 \%)$ \\
\hline $\begin{array}{l}\text { Distal LM with ostial LAD and ostial } \\
\text { LCX }\end{array}$ & $8(5.22 \%)$ \\
\hline Distal LM and Ramus & $2(1.3 \%)$ \\
\hline
\end{tabular}

Outcomes of the study: There was no mortality in the study but on table stent thrombosis was seen in one patient for which thrombuster was used, IABP was placed immediately and sent for CABG. For two cases stent could not be negotiated because of heavy calcification so POBA was done and for one case after deployment and post dilatation of stent the result was suboptimal but flow was TIMI III. 


\section{DISCUSSION:}

Many studies have shown that stenosis of the LMCA is of critical prognostic importance. [10-14]. Observational studies of medically $[9,15]$ and surgically [16-18]. Treated patients with LMCA disease support the widely accepted belief that, in general, CABG surgery lessens symptoms and significantly prolongs survival. More importantly, observational $[19,20]$ and randomized [15, $21,22]$. Studies designed to directly compare surgical and medical therapy in patients with left main stenosis have confirmed the benefits of surgical revascularization, although in all but two of the randomized studies [12-23] follow-up was less than 4 years. Recent studies have now supported the use of angioplasty and drug eluted stents in "unprotected and protected angioplasty" of the left main artery as an alternative to CABG [24,25]. With improved stenting techniques and the availability of DESs, many institutions have adopted stent treatment of unprotected LM disease as a more routine strategy. In addition, at many centers the threshold for favoring stenting over CABG for LM disease has also been lowered.

The choice of PCI or CABG for treatment of unprotected LMCA disease depends on several clinical and anatomic features, making optimal patient selection crucial for appropriate treatment of LMCA disease and achievement of favorable long-term outcomes $[27,28]$. In patients with very complex anatomic features, which are not feasible for stenting, and concomitant diffuse multivessel disease, CABG is preferred so as to avoid procedural and future thrombotic risks and to provide more complete revascularization. However, in patients with relatively simple LMCA disease, such as ostial/ shaft LMCA disease or isolated LMCA disease (with or without one or 2-vessel involvement), PCI is an alternative, and in some cases a preferred strategy to reduce surgical risks (e.g., stroke and in-hospital events following major surgery).LMCA lesion characteristics (severe calcification, distal LMCA involvement with relation to major branches), the extent of extra-LMCA (concomitant multi vessel disease, the status of distal run-off), and patient clinical characteristics (age, diabetes, ejection fraction, and other co morbidities) are important in patient selection.
Briefly, selection of patients for PCI may be optimized as follows: (1) PCI with stenting is a reasonable option for patients with unprotected LMCA disease at high surgical risk or with protected LMCA disease; (2) patients presenting with acute coronary syndrome who have culprit LMCA occlusion and hemodynamic instability requiring emergent revascularization; and (3) isolated ostial or mid shaft LMCA disease. For patients with clinical and anatomic characteristics suitable for both CABG and PCI, the benefits and risks of PCI versus CABG and patient/ physician preference, need to be weighted.

Balloon angioplasty of unprotected LMCA stenosis has been associated with varying degrees of procedural success, but generally poor long-term prognosis [29-32]. Previously reported unprotected LMCA stent procedures have been done in conditions of prohibitive surgical risk or in bailout situations. Other investigators have reported primarily protected LMCA stenting [3335].

This clinical study shows that stenting of unprotected and protected LMCA stenosis improves the immediate procedural success and is safe and effective. Takagi and colleagues ${ }^{[45]}$ reported a 3 year cardiac mortality rate of $4.2 \%$ in low risk patients but our study showed no mortality. As observed in other studies, ${ }^{[46-48]}$ in the present study the angiographic success rate during angioplasty was $98.6 \%$ there was one episode of acute stent thrombosis $(0.65 \%)$. out of total unprotected cases $80 \%$ are females and $65 \%$ are males with $\mathrm{P}$ value significance $(\mathrm{P}=0.05)$.

The outcome of such protected left main interventions is more favorable than when no patent graft to the left coronary system is present [48-50]. Here in this study we found no significant difference in outcome between protected and unprotected LMCA angioplasty.

These favorable outcomes and procedural success had possible explanations such as presence of low percentage of risk factors like hypertension (15\%), diabetes $(15 \%)$, mean age of 58yrs. LV dysfunction present in one third of cases only out of which severe LV dysfunction in $6 \%$ males and in $15 \%$ females only. Balloon inflations were kept $<60 \mathrm{sec}$ in duration. There was significant difference in clinical presentation between males and females like $74.6 \%$ of males presented with ACS (acute coronary syndrome) where 
as $25 \%$ females with ACS. Regardless of anticoagulation therapy, major in-hospital complications did not occur in the current study. This finding suggests that antiplatelet therapy alone might be an effective poststent antithrombotic regimen even in the LMCA.

Ostial and mid shaft lesions were managed as stenting in other main coronary arteries, but distal bifurcation lesions had some sorts of technical challenges. The technique for bifurcation lesions included stenting of the left main artery into a major branch of the left coronary artery with or without stenting of the other branch, depending on the degree of residual stenosis of the other branch (e.g., left anterior descending or left circumflex artery).

In our study 56 patients had distal LMCA lesion. Out of 56 cases, 48 cases had significant ostial disease of either LAD, Ramus or LCX which were managed with single stent strategy. Eight distal bifurcation lesions were managed with double stent strategy with final kissing. All stents were implanted with moderate to high deployment pressure (10-16 Atm) followed by systematic high pressure (16-20 Atm) after dilatation with a non-compliant balloon.

FFR was used in 2 cases and ostial flaring in 5 cases. Currently available evidence suggest that results are less favorable when distal LMCA lesions are treated by a 2stent approach compared with single-stent approach. The TLR rate is relatively low (5\%) with single stent approaches, even for distal LMCA lesions, and is nearly equivalent to results obtained with DES for ostial or mid-left main lesions. However, patients with distal LMCA lesions treated with 2-stent techniques showed a TLR rate as high as $25 \%$, with restenosis confined mainly to the left circumflex ostium. one patient developed peri-procedural LMCA thrombosis for which thrombuster was used, IABP was placed and sent for CABG. IABP was not used prophylactically in any case. Rotational atherectomy before LMCA stenting was performed in two patients but stent could not be negotiated so POBA(plain old balloon angioplasty) was done with optimal result. The beneficial effects of the debulking procedure before LMCA stenting needs to be evaluated in larger numbers of patients. However, preliminary data support the concept of debulking before stenting [36,37].
Park et al performed PTCA with stent deployment in unprotected LMCA in 42 patients with preserved left ventricular function. Forty one of these patients refused surgery. Their results were encouraging, achieving immediate success in all cases. After six months, the re stenosis rate was $22 \%$. The only death occurred two days after revascularization surgery in a patient with intrastent restenosis. Some reports have also shown that in impending occlusion of the LMCA in unstable patients this procedure can be lifesaving, avoiding the risks of coronary surgery. Even more complex lesions, such as LMCA bifurcation can be treated with success and immediate improvement in the patient. Indeed, procedural mortality was $9.1 \%$ in a study by O'Keefe et al [38] and $12 \%$ in a study by Eldar et al [39].

Our results showed good immediate results with zero mortality. Stenting reduces the need for hemodynamic assistance (intra aortic counter pressure balloon, circulatory support), which is a well established source of local morbidity[40] and rapid stent delivery ensures an optimal result without prolonged ischemia. The present study did not include angiographic follow-up.

In patients with LMCA stenting, it is advisable to monitor their clinical condition regularly during the first few months after angioplasty. In addition, it has been suggested that angiographic studies should be carried out early, between 6 and 16 weeks following angioplasty, to detect rapidly developing restenosis [42]. However, the actual benefit of angiography in this context has not been established. The use of DES could help reduce cardiac event and revascularization rates during follow up $[42,44]$.

In the present study $80 \%$ of the patients received DES(drug eluting stents) The main limitations of the present study are that it was a non-randomized study and that there was no angiographic follow-up.

We assume that when PCI is performed by experienced hands, its technical feasibility and safety is no longer of major concern. The procedure can relieve most LMCA obstructions with very good immediate results. Nevertheless, the durability of the results and the survival benefit of PCI compared to surgery remain important concerns.

Our study shows excellent procedural success rate (98\%) with an excellent overall in-hospital survival rate (100\%). 
Main limitation of this study was lacking of IVUS to assess exact placement and optimal expansion of the stent which is especially important for distal bifurcation lesion to ensure adequate coverage of the lesion. Despite this limitation our study shows that stenting of unprotected LMCA stenosis is a safe and effective revascularization procedure.

\section{CONCLUSIONS:}

This study which investigated the safety and feasibility of coronary stenting in patients with significant LMCA stenosis yielded several striking clinical implications. Present study demonstrated that stenting for significant LMCA disease is safe and feasible with very high procedural and clinical success rates and zero mortality. Despite clear recommendation of CABG as the gold standard in the treatment of significant LMCA disease, Our findings, highlight the reliability of PCI as a safe and feasible therapeutic option for patients with significant LMCA disease.

\section{REFERENCES:}

1.Caracciolo EA, Davis KB, Sopko G, et al. Comparison of surgical and medical group survival in patients with lef main equivalent coronary artery disease. Longterm CASS experience. Circulation 1995; 91: 2335-44.

2. O'Keefe JH Jr, Hartzler GO, Rutherford BD, et al. Lef main coronary angioplasty: early and late results of 127 acute and elective procedures. Am J Cardiol 1989; 64: 144-7.

3. Silvestri M, Barragan P, Sainsous J, et al. Unprotected lef main coronary artery stenting: immediate and medium-term outcomes of 140 elective procedures. J Am Coll Cardiol 2000; 35: 1543-50.

4. Tan WA, Tamai H, Park SJ, et al; ULTIMA Investigators. Long-term clinical outcomes after unprotected lef main trunk percutaneous revascularization in 279 patients. Circulation 2001; 104: 1609-14.

5. Takagi T, Stankovic G, Finci L, et al. Results and longterm predictors of adverse clinical events after elective percutaneous interventions on unprotected lef main coronary artery. Circulation 2002; 106: 698-702.
6. Black A, Cortina R, Bossi I, Choussat R, Fajadet J, Marco J. unprotected LCA stenting. Correlates of midterm survival and impact of patient selection. J Am Coll Cardiol 2001;37:832-8.

7. Colombo A, Hall P, Nakamura S; Intracoronary stenting without anticoagulation accomplished with intravascular ultrasound guidance. Circulation. 91 1995: 1676-1688.

8. Schoemig A, Neumann FJ, Kastrati A; A randomized comparison of antiplatelet and anticoagulant therapy after the placement of coronary artery stents. N Engl J Med. 334 1996; 1084-1089.

9. DeMots H, Rosch J, McAnulty JH, et al. Left main coronary artery disease. Cardiovasc Clin 1977;8:201-11.

10. Varnauskas E, for the European Coronary Surgery Study Group. Twelve-year follow-up of survival in the randomized European Coronary Surgery Study. N Engl J Med 1988;319:332-7.

11. The Veterans Administration Coronary Artery Bypass Surgery Cooperative Study Group. Eleven-year survival in the Veterans Administration randomized trial of coronary bypass surgery for stable angina. $\mathrm{N}$ Engl J Med 1984;311:1333-9.

12. European Coronary Surgery Study Group. Longterm results of a prospective randomized study of coronary artery bypass surgery in stable angina pectoris. Lancet 1982;2:1173-80.

13. European Coronary Surgery Study Group. Prospective randomized study of coronary artery bypass surgery in stable angina pectoris: second interim report. Lancet 1980;2:491-5.

14. Detre KM, Murphy ML, Hultgren HN. Effect of coronary bypass surgery on longevity in high and low risk patients: report from the VA Cooperative Coronary Surgery Study. Lancet 1977;2:1243-5.

15. Lim JS, Proudfit WL, Sones FM Jr. Left main coronary arterial obstruction: long term follow-up of 141 non-surgical cases. Am J Cardiol 1975;36:131-5. 
16. Demots H, Bonchek LI, Rosch J, et al. Left main coronary artery disease: risks o 182 Curr Probl Cardiol, March 2007 angiography, importance of coexisting disease of other coronary arteries and effects of revascularization. Am J Cardiol 1975;36:136-41.

17. Alford WC Jr, Shaker IJ, Thomas CS Jr, et al. Aortocoronary bypass in the treatment of left main coronary artery stenoses. Ann Thorac Surg 1974;17:247-53.

18. Zeft JH, Manley JC, Huston JH, et al. Left main coronary artery stenosis: results of coronary bypass surgery. Circulation 1974;49:68-76.

19. Talano JV, Scanlon PJ, Meadows WR, et al. Influence of surgery on survival in 145 patients left main coronary artery disease. Circulation 1975;51(suppl I):I-105-I-111.

20. Cohen MV, Gorlin R. Main left coronary artery disease: clinical experience from 1964-1974. Circulation 1975;52:275-85.

21 . Hultgren HN, Detre KM, Takaro T, et al. The VA cooperative study of coronary arterial surgery: baseline characteristics of study population and survival in subgroup with medical versus surgical treatment. Prog Cardiol 1977;6:67-81.

22. Takaro $\mathrm{T}$, Hultgren $\mathrm{HN}$, Lipton $\mathrm{MJ}$, et al, for Participants in the Study Group. The VA Cooperative Randomized Study of surgery for coronary arterial occlusive disease, II: subgroup with significant left main lesions. Circulation 1976;54 (suppl III):III-107-III-117.

23. Kosuga $\mathrm{K}$, Tamai $\mathrm{H}$. Left main stem coronary disease: the case for percutaneous coronary intervention in a high risk patient with complex disease. Heart 2005; 91(suppl 3):iii35-iii38.

24. Park SJ, Park SW, Hong MK, et al. Long-term (threeyear) outcomes after stenting of unprotected left main coronary artery stenosis in patients with normal left ventricular function. Am J Cardiol 2003;91:12-6.

25. Karam C, Fajadet J, Cassagneau B, et al: Results of stenting of unprotected left main coronary artery stenosis in patients at high surgical risk. Am J Cardiol 1998; 82:975-8.

26. Ormiston JA, Stewart FM, Roche AHG, et al. Late regression of the dilated site after coronary angioplasty. A 5-year quantitative angiographic study. Circulation 1997;96:468

27. Seung KB, Park DW et al. Stents versus coronaryartery bypass grafting for left main coronary artery disease. N Engl J Med. 2008;358:1781-1792.

28. Park SJ, Park DW. Drug-eluting stents for left main coronary artery stenosis: case selection and technical issues. Am Heart Hosp J. 2008;6:21-29.

29. Gruentzig AR, Senning A, Siegenthaler WE. Non operative dilatation of coronary artery stenosis. N Engl J Med 1979;301:61-7.

30. O'Keefe JH, Hartzler GO, Rutherford BD, et al. Left main coronary angioplasty: early and late results of 127 acute and elective procedures. Am J Cardiol 1989;64:144-7.

31. Hartzler GO, Rutherford BD, McConohay DR, Johnson WL, Giorgi LV. "High-risk" percutaneous transluminal coronary angioplasty. Am J Cardiol 1988;61:33G-37G.

32. Eldar M, Schulhoff RN, et al. Results of percutaneous transluminal coronary angioplasty of the left main coronary artery. Am J Cardiol 1991;68:255-6

33. Tamura T, Nobuyoshi M, Nosaka H, et al. PalmazSchatz stenting in unprotected and protected left main coronary artery: immediate and follow-up result [abstract]. Circulation 1996;94 Suppl I:I-671.

34. Ellis SG, Moses J, White HJ, et al. Contemporary percutaneous treatment of unprotected left main stenosis: a preliminary report of the ULTIMA(Unprotected Left Main Trunk Intervention Multicenter Assessment) registry [abstract]. Circulation 1996;94 Suppl I:I-671.

35. Karam C, Jordan C, Fajadet J, Cassaneau B, Laurent JP, Marco J. Six-month follow-up of unprotected left 
main coronary artery stenting [abstract]. Circulation 1996;94 Suppl I:I-627.

36. Moussa I, Moses J, Di Mario C, King T, Reimers B, Colombo A. Immediate and short-term results of the pilot phase of stenting after optimal lesion debulking: 'the SOLD trial' [abstract]. J Am Coll Cardiol 1997;29 Suppl: 415A.

37. Hoffmann R, Mintz GS, Kent KM, et al. Is there an optimal therapy for calcified lesions in large vessels? Comparative acute and follow-up results of rotational atherectomy, stents, or the combination[abstract]. J Am Coll Cardiol 1997;29 Suppl:68-9A.s

38. O'Keefe JH, Hartzler GO, Rutherford BD, et al. Left main coronary angioplasty: early and late results of 127 acute and elective procedures. Am J Cardiol 1989;64:144-7

39. Eldar M, Schulhoff RN, Hertz I, Frankel R, Feld H, ShaniJ. Results of percutaneous transluminal coronary angioplasty of the left maincoronary artery. Am J Cardiol 1991;68:255-6.

40. Tommaso CL, Vogel JHK. Coronary angioplasty in high risk patients with left main coronary stenosis: results from the national registry of elective supported angioplasty. Cathet Cardiovasc Diagn1992;25:169-73.

41. Ellis SG, Tamai H, Nobuyoshi $M$, et al. Contemporary percutaneous treatment of unprotected left main coronary stenoses. Initial results from a multicenter registry. Analysis 1994-1996. Circulation 1997;96:3867-72.

42. Tan WA, Tamai H, Park SJ, Plokker T, Nobuyoshi M, Suzuki $\mathrm{T}$, et al. Long-term outcomes after unprotected left main trunk percutaneous revascularization in 279 patients. Circulation 2001; 104:1609-14.

43. Arampatzis CA, Lemos PA, Hoye A, Degertakin M, Saia $\mathrm{F}$, Lee $\mathrm{CH}$, et al. Effectiveness of sirolimus-eluting stent for treatment of LCA disease. Am J Cardiol 2003;92:327-9.

44. Morice MC, Serruys PW, Sousa JE, Fajadet J, Ban Hayashi E, Perin M, et al. A randomized comparison ofsirolimus-eluting stent with a standard stent for coronary revascularization. N Engl J Med 2002;346:177380 .

45. Takagi T, Stankovic G, Finci L, et al. Results and long-term predictors of adverse clinical events afer elective percutaneous interventions on unprotected lef main coronary artery. Circulation 2002; 106: 698-702.

46. López JJ, Ho KKL, Stoler RC, Caputo RP, Carrozza JP, Kuntz R, et al. Percutaneous treatment of protected and unprotected left main coronary stenoses with new devices: immediate angiographic results and intermediate-term follow-up. J Am Coll Cardiol 1997;29:345-52.

47. Silvestri M, Barragan P, Sainsous J, Bayet G, Simeoni JB, Roquebert PO, et al. Unprotected LCA stenting: Immediate and medium term outcomes of 140 elective procedures. J Am Coll Cardiol 2000;35:1543-50.

48.. Kornowski R, Klustein M, Satler LF, et al. Impact of stents on clinical outcomes in percutaneous left main coronary artery revascularization. Am J Cardiol 1998; 82:32-7.

49. Keeley EC, Aliabadi D, O'Neill WW, et al. Immediate and long-term results of elective and emergent percutaneous interventions on protected and unprotected severely narrowed left main coronary arteries. Am J Cardiol 1999;83:242-6

50. Hong M-K, Mintz GS, Hong MK, et al. Intravascular ultrasound predictors of target lesion revascularization after stenting of protected left main coronary artery stenosis. Am J Cardiol 1999;83:175. 